\title{
CANONICAL MEASURE ASSIGNMENTS
}

\author{
STEVE JACKSON AND BENEDIKT LÖWE
}

\begin{abstract}
We work under the assumption of the Axiom of Determinacy and associate a measure to each cardinal $\kappa<\aleph_{\varepsilon_{0}}$ in a recursive definition of a canonical measure assignment. We give algorithmic applications of the existence of such a canonical measure assignment (computation of cofinalities, computation of the Kleinberg sequences associated to the normal ultrafilters on all projective ordinals).
\end{abstract}

\section{INTRODUCTION}

One of the striking features of set theory under the Axiom of Determinacy is the fact that there is a full analysis of the cardinal structure for a fairly large initial segment of $\Theta:=\sup \{\alpha$ : there is a surjection from $\mathbb{R}$ onto $\alpha\}$, something which we cannot hope to get in the ZFC context. While almost none of the combinatorial properties of small cardinals (e.g., $\aleph_{2}, \aleph_{3}, \aleph_{\omega^{2}}$ ) are fixed in ZFC, ZF + AD gives us definite combinatorial properties (e.g., measurability, Jónssonness, Rowbottomness) of these cardinals, in particular below $\aleph_{\varepsilon_{0}}$, the supremum of the projective ordinals (defined in $\S 2$ ).

This structure is closely tied to an analysis of measures on the projective ordinals and the representation of cardinals as ultrapowers via these measures. In [Ja99] this analysis is given below $\boldsymbol{\delta}_{5}^{1}$, and in [Ja88] it is extended to all the $\boldsymbol{\delta}_{n}^{1}$. A key combinatorial ingredient in this analysis is the notion of a description, which give a precise presentation of the cardinal structure (see also [Ja $\infty$ ] for an introduction to this theory). In $[\mathrm{JaKh} \infty]$ it was shown that a certain fairly simple family of measures on $\boldsymbol{\delta}_{3}^{1}$ could be used to directly describe the cardinal structure below $\boldsymbol{\delta}_{5}^{1}$. This presentation of the cardinal structure avoided the notion of description, although the description theory was an integral part of the proofs.

Our goal here is to present a simple combinatorial framework which suffices to describe the cardinal structure below the supremum of the projective ordinals, and which also avoids the description analysis. We introduce the notion of an ordinal algebra, and we inductively assign measures to the elements of this algebra through two lifting operations. This gives us a comparitively simple notational framework for describing the cardinal structure below the $\boldsymbol{\delta}_{n}^{1}$ which is of independent interest and will also allow those not familiar with the description analysis to use many of the strong consequences of that theory.

We emphasize that we do not prove here the strong partition relation on the $\boldsymbol{\delta}_{2 n+1}^{1}$ (although we use it heavily), nor do we prove that the ultrapowers of the

2000 Mathematics Subject Classification. 03E60 03E55 03E05.

The first author was supported by NSF Grant DMS-0097181; the second author's visits to Denton TX were made possible by a DFG-NWO Bilateral Cooperation Grant (DFG KO 1353/31, NWO 61-532), an NWO reisbeurs (R-62-616) and the Roy McLeod Millican Memorial Fund. 
$\delta_{2 n+1}^{1}$ by the measures we construct have the correct values. Rather, we abstract these assumptions into a "canonicity assumption" (defined precisely later) from which our analysis proceeds. The proof of this assumption below $\boldsymbol{\delta}_{5}^{1}$ is given in $[\mathrm{JaKh} \infty]$, and for the general case will appear later (although the description analysis necessary is given in [Ja88]).

In this paper we would like to stress the algorithmic nature of this analysis of the cardinals below $\aleph_{\varepsilon_{0}}$ : we describe a general recursive procedure of measure assignment $(\S 5)$, and develop algorithms for

- computing all regular cardinals below $\aleph_{\varepsilon_{0}}(\S 6.1)$

- computing the cofinalities of all cardinals below $\aleph_{\varepsilon_{0}}(\S 6.2)$, and

- computing the Kleinberg sequences derived from all normal measures on the projective ordinals $(\S 6.3)$

under the assumption that the measure assignment is canonical.

\section{Mathematical Background.}

In this paper, we shall be working in the theory $Z F+D C+A D$. We shall say that a cardinal $\kappa$ has the strong partition property if the partition relation $\kappa \rightarrow(\kappa)^{\kappa}$ holds, i.e., if for every partition of $[\kappa]^{\kappa}$ into two blocks there is a homogeneous set of order type $\kappa$. We say that it has the weak partition property if for all $\alpha<\kappa$, the partition relation $\kappa \rightarrow(\kappa)^{\alpha}$ holds. Note that the strong and the weak partition properties cannot hold for any uncountable cardinal if we assume the Axiom of Choice AC: by a result of Erdős and Rado (cf. [Ka94, Proposition 7.1]) any partition relation with infinite exponents violates AC.

In practice, we actually use an equivalent variation of these definitions, which the reader can take as our official definition We first recall some terminology.

Let $\alpha$ and $\kappa$ be ordinals. A function $f: \alpha \rightarrow \kappa$ is continuous if and only if for all limit ordinals $\gamma<\alpha$,

$$
f(\gamma)=\sup \{f(\xi) ; \xi<\gamma\} .
$$

The function $f$ has uniform cofinality $\omega$ if there is a function $h: \omega \times \alpha \rightarrow \kappa$, which is increasing in the first argument, such that for $\gamma<\alpha$, we have

$$
f(\gamma)=\sup \{h(n, \gamma): n \in \omega\} .
$$

We say a function $f: \alpha \rightarrow \kappa$ is of the correct type if it is increasing, everywhere discontinuous (i.e., for all $\gamma<\alpha, f(\gamma)>\sup _{\beta<\gamma} f(\beta)$ ), and of uniform cofinality $\omega$. We say $f: \alpha \rightarrow \kappa$ is of continuous type if it is increasing, continuous and has uniform cofinality $\omega$ at all successor ordinals (with obvious meaning).

We can now write $\kappa \stackrel{\text { club }}{\longrightarrow}(\kappa)^{\lambda}$ for the statement "for every partition $\mathcal{P}$ of the functions from $\lambda$ to $\kappa$ of the correct type into two sets there is a club set $C \subseteq \kappa$ such that all functions $f: \lambda \rightarrow C$ of the correct type get the same color by $\mathcal{P}$ ". It is easy to see that if $\lambda=\omega \cdot \lambda$, then $\kappa \rightarrow(\kappa)^{\lambda}$ and $\kappa \stackrel{\text { club }}{\longrightarrow}(\kappa)^{\lambda}$ are equivalent ( $c f$. [Ja99, p. 5] or [Ja $\infty$, Fact 2.28]) and so we can freely switch between the two definitions for the weak and strong partition properties.

For $\lambda<\kappa, \lambda$ regular, let us define the $\lambda$-cofinal filter $\mathcal{C}_{\kappa}^{\lambda}$ as the filter generated by the $\lambda$-closed unbounded sets in $\kappa$, i.e.,

$$
A \in \mathcal{C}_{\kappa}^{\lambda}: \Longleftrightarrow \text { there is a club set } C \subseteq \kappa \text { such that }\{\alpha \in C: \operatorname{cf}(\alpha)=\lambda\} \subseteq A \text {. }
$$


Clearly, $\mathcal{C}_{\omega_{1}}^{\omega}$ is the ordinary club filter on $\omega_{1}$. As usual, we call $\sigma$-complete ultrafilters on $\kappa$ measures, we call a measure normal if it is closed under diagonal intersection and semi-normal if it contains all club subsets of $\kappa$. If $\mu$ is a measure on $\varrho$ and $\alpha$ is an ordinal, then (because of DC) the ultrapower $\alpha^{\varrho} / \mu$ is wellfounded and thus isomorphic to an ordinal. We identify it with its Mostowski collapse. We call a cardinal $\kappa$ closed under ultrapowers if for all $\varrho<\kappa$ and all measures $\mu$ on $\varrho$, we have that $\varrho^{\varrho} / \mu<\kappa$. If $\kappa$ is regular, this is equivalent to the statement "for all $\varrho<\kappa$ and all measures $\mu$ on $\varrho$, we have that $\kappa^{\varrho} / \mu=\kappa "$.

The weak partition property of $\kappa$ implies the existence of many concrete measures on $\kappa$, as the following theorem of Kleinberg shows:

Theorem 1. Let $\kappa$ be a cardinal with the weak partition property and $\lambda<\kappa$ a regular cardinal. Then $\mathcal{C}_{\kappa}^{\lambda}$ is a normal measure. In addition, if $\kappa$ is not weakly Mahlo, then these are the only normal ultrafilters on $\kappa$.

Proof. [Ka94, Theorem 28.10 \& Exercise 28.11].

In other words, the weak partition property of $\kappa$ not only gives the existence of measures, but in our case (our cardinals will be below $\aleph_{\varepsilon_{0}}$ and thus not weakly Mahlo) also a structured pattern of all of the normal measures on $\kappa$ (indexed by the regular cardinals below $\kappa$ ).

In addition, the strong partition property also connects to other combinatorial properties:

Definition 2. Let $\kappa$ be a strong partition cardinal and $\mu$ a normal measure on $\kappa$. We then define a sequence $\left\langle\kappa_{n}^{\mu}: n<\omega\right\rangle$ as follows:

- $\kappa_{0}^{\mu}:=\kappa$,

- $\kappa_{n+1}^{\mu}:=\left(\kappa_{n}^{\mu}\right)^{\kappa} / \mu$, and

This sequence is called the Kleinberg sequence derived from $\mu$.

Theorem 3. Let $\kappa$ be a strong partition cardinal and $\mu$ be a measure on $\kappa$. Then $\kappa^{\kappa} / \mu$ is a cardinal.

If $\mu$ is normal, then $\kappa_{1}^{\mu}=\kappa^{\kappa} / \mu$ is a measurable cardinal, and all $\kappa_{n}^{\mu}$ are Jónsson cardinals.

Proof. The first claim is a result of Martin's proved in [Ja99, Theorem 7.1]. The second claim is part of Kleinberg's analysis of strong partition cardinals from [Kl77].

The projective ordinals play an important role in the descriptive set theory of the projective sets $(c f .[\mathrm{Mo} 80, \S 7 \mathrm{D}],[\mathrm{Ka} 94, \S 30]$, and [Ke78]). Moreover, the fact that the odd projective ordinals $\boldsymbol{\delta}_{2 n+1}^{1}$ have the strong partition property is central to the analysis of cardinals and measures below thier supremum. Recall they are define by:

$$
\boldsymbol{\delta}_{n}^{1}:=\sup \left\{\xi: \xi \text { is the length of a prewellordering of } \omega^{\omega} \text { in } \boldsymbol{\Delta}_{n}^{1}\right\} .
$$

The Cabal has developed an intricate theory of the combinatorics of the projective ordinals summarized in the following fact:

Theorem 4. Let $n$ be a natural number. Then:

(1) (Kunen, Martin 1971) $\boldsymbol{\delta}_{2 n+2}^{1}=\left(\boldsymbol{\delta}_{2 n+1}^{1}\right)^{+}$, 
(2) (Kunen, Martin 1971) all $\boldsymbol{\delta}_{n}^{1}$ are measurable,

(3) (Kunen, Martin, Solovay 1971) $\boldsymbol{\delta}_{2}^{1}=\aleph_{2}, \boldsymbol{\delta}_{3}^{1}=\aleph_{\omega+1}$, and $\boldsymbol{\delta}_{4}^{1}=\aleph_{\omega+2}$,

(4) (Martin 1971) $\boldsymbol{\delta}_{1}^{1} \rightarrow\left(\boldsymbol{\delta}_{1}^{1}\right)^{\boldsymbol{\delta}_{1}^{1}}$

(5) (Kechris 1974) for all $n, \boldsymbol{\delta}_{2 n+1}^{1}$ is a successor of a cardinal of cofinality $\omega$,

(6) (Kunen 1971) the $\omega$-cofinal measure $\mathcal{C}_{\delta_{2 n+1}^{1}}^{\omega}$ is a normal measure on $\boldsymbol{\delta}_{2 n+1}^{1}$ with $\boldsymbol{\delta}_{2 n+1}^{1} \boldsymbol{\delta}_{2 n+1}^{1} / \mathcal{C}_{\boldsymbol{\delta}_{2 n+1}^{1}}^{\omega}=\boldsymbol{\delta}_{2 n+2}^{1}=\left(\boldsymbol{\delta}_{2 n+1}^{1}\right)^{+}$,

(7) (Jackson, Martin 1980) $\boldsymbol{\delta}_{3}^{1} \boldsymbol{\delta}_{3}^{1} / \mathcal{C}_{\boldsymbol{\delta}_{3}^{1}}^{\omega_{1}}=\aleph_{\omega \cdot 2+1}$ and $\boldsymbol{\delta}_{3}^{1} \boldsymbol{\delta}_{3}^{1} / \mathcal{C}_{\delta_{3}^{1}}^{\omega_{2}}=\aleph_{\omega^{\omega}+1}$, and these two cardinals are measurable.

(8) (Jackson 1985) Let $\mathbf{e}_{0}:=0$ and $\mathbf{e}_{n+1}:=\omega^{\left(\omega^{\mathbf{e}_{n}}\right)}$ (i.e., $\mathbf{e}_{n}$ is a exponential $\omega$-tower of height $2 n-1)$. Then for every $n \in \omega$,

$$
\delta_{2 n+1}^{1}=\aleph_{\mathbf{e}_{n}+1},
$$

and all odd projective ordinals have the strong partition property and are closed under ultrapowers.

Proof. A proof of all parts except for the last two can be found in [Ke78]. Item (7) and the $n=2$ case of (8) can be found in [Ja99, Chapter 7]. The general case of (8) is in [Ja88].

\section{OrdinAl ALGEBRAS AND MEASURE ASSIGNMENTS}

An ordinal algebra is a free algebra $\mathfrak{A}$ over a set of generators $\mathfrak{V}=\left\{\mathrm{V}_{\beta}\right\}_{\beta<\alpha}$ using the binary operations $\oplus, \otimes$. We write $\mathfrak{A}_{\alpha}$ for the algebra with $\alpha$ generators. For $\alpha<\beta$ we naturally have $\mathfrak{A}_{\alpha} \subseteq \mathfrak{A}_{\beta}$. For any ordinal algebra $\mathfrak{A}$ we define a function $o$ from $\mathfrak{A}$ onto an ordinal ht $(\mathfrak{A})$ which we call the height of $\mathfrak{A}$. We will have for $\alpha<\beta$ that the $o$ function on $\mathfrak{A}_{\beta}$ extends the $o$ function on $\mathfrak{A}_{\alpha}$. To begin, we define $o\left(\mathrm{~V}_{0}\right)=0$. Suppose we have defined $o$ on $\mathfrak{A}_{\alpha}$. Then set $o\left(\mathrm{~V}_{\alpha}\right)=$ $\operatorname{ht}\left(\mathfrak{A}_{\alpha}\right)=\sup \left\{o(t)+1: t \in \mathfrak{A}_{\alpha}\right\}$. Then extend $o$ to $\mathfrak{A}_{\alpha+1}$ by $o(s \oplus t)=o(s)+o(t)$, $o(s \otimes t)=o(s) \cdot o(t)$ (ordinal addition and multiplication). By construction, $o$ is a homomorphism from the free algebra to the ordinals with ordinal addition and multiplication.

Let us look at the simplest ordinal algebras as an example. For this, we introduce a notation for finitely iterated sums and products:

$$
\begin{aligned}
\mathrm{V} \otimes n & :=\mathrm{V} \underbrace{\oplus \ldots \oplus}_{n} \mathrm{~V}, \text { and } \\
\mathrm{V}^{\otimes n}: & =\mathrm{V} \underbrace{\otimes \ldots \otimes}_{n} \mathrm{~V} .
\end{aligned}
$$

- $(\alpha=1)$. If $\mathfrak{V}=\left\{\mathrm{V}_{0}\right\}$, then $o\left(\mathrm{~V}_{0}\right)=0$, so $o\left(\mathrm{~V}_{0} \oplus \mathrm{V}_{0}\right)=0, o\left(\mathrm{~V}_{0} \otimes \mathrm{V}_{0}\right)=0$, etc., so ht $(\mathfrak{A})=1$.

- $(\alpha=2)$. If $\mathfrak{V}=\left\{\mathrm{V}_{0}, \mathrm{~V}_{1}\right\}$, then $o\left(\mathrm{~V}_{0}\right)=0$ and $o\left(\mathrm{~V}_{1}\right)=1$, so $o\left(\mathrm{~V}_{1} \otimes n\right)=n$, and thus ht $(\mathfrak{A})=\omega$.

- $(\alpha=\omega)$. Here we use $\omega$ generators $\mathfrak{V}=\left\{\mathrm{V}_{0}, \bigvee_{1}, \bigvee_{2}, \ldots\right\}$. So, $o\left(\bigvee_{0}\right)=0$, $o\left(\mathrm{~V}_{1}\right)=1$, and $o\left(\mathrm{~V}_{2}\right)=\omega$. Then $o\left(\mathrm{~V}_{2}^{\otimes n}\right)=\omega^{n}$, and thus $o\left(\mathrm{~V}_{3}\right)=\omega^{\omega}$. Likewise, $o\left(\bigvee_{4}\right)=\omega^{\omega^{2}}, o\left(\bigvee_{5}\right)=\omega^{\omega^{3}}$, etc. So, ht $\left(\mathfrak{A}_{\omega}\right)=\omega^{\omega^{\omega}}$. 


$$
T_{x} \quad T_{y} \quad T
$$

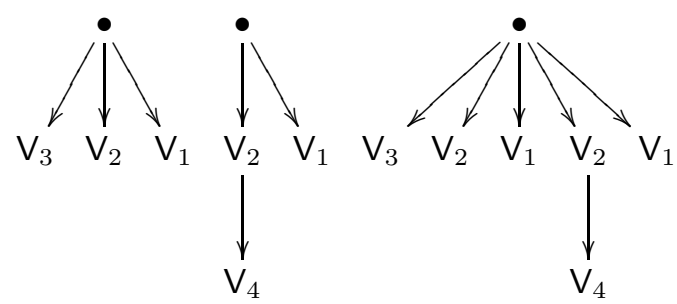

Figure 1. Adding the trees for $x=\mathrm{V}_{3} \oplus \mathrm{V}_{2} \oplus \mathrm{V}_{1}$ and $y=$ $\left(\mathrm{V}_{4} \otimes \mathrm{V}_{2}\right) \oplus \mathrm{V}_{1}$.

\section{Proposition 5.}

$$
o\left(\mathrm{~V}_{\alpha}\right)=\operatorname{ht}\left(\mathfrak{A}_{\alpha}\right)=\left\{\begin{array}{cl}
1 & \alpha=1 \\
\omega^{\omega^{\alpha-2}} & 1<\alpha<\omega \\
\omega^{\omega^{\alpha}} & \alpha \geq \omega .
\end{array}\right.
$$

Proof. An easy induction on $\alpha$. Suppose that ht $\left(\mathfrak{A}_{\alpha}\right)=\omega^{\omega^{\alpha}}$. By definition, $o\left(\mathrm{~V}_{\alpha}\right)=$ $\omega^{\omega^{\alpha}}$. Also, $o\left(\bigvee_{\alpha}^{\otimes n}\right)=\left(\omega^{\omega^{\alpha}}\right)^{n}=\omega^{\omega^{\alpha} \cdot n}$. So,

$$
\operatorname{ht}\left(\mathfrak{A}_{\alpha+1}\right)=\sup _{n} \omega^{\left(\omega^{\alpha} \cdot n\right)}=\omega^{\omega^{\alpha+1}} .
$$

Let us fix an ordinal algebra $\mathfrak{A}$ with set of generators $\mathfrak{V}$.

In the following, we shall identify terms in an ordinal algebra with finite labelled ordered trees $\langle T, \ell\rangle$. We assume that there is an implicit order on the set of immediate successors of a node that we read from left to right in the pictures. All of our trees have a root $\bullet$ and the labelling function $\ell$ is a map from $T \backslash\{\bullet\}$ into $\mathfrak{V}$. When convenient, we may assume without loss of generality that $T$ is a finite subtree of $\omega^{<\omega}$, that is, the nodes of $T$ can be viewed as finite sequences $\left\langle i_{0}, \ldots, i_{k}\right\rangle$ of integers. We recursively associate a tree to each term in $\mathfrak{A}$ :

(1) We identify the variable $v$ with the tree consisting of a root $\bullet$ and one immediate successor node $v$ such that $\ell(v):=\mathrm{v}$.

(2) If $x, y \in \mathfrak{A}$ are represented by $\left\langle T_{x}, \ell_{x}\right\rangle$ and $\left\langle T_{y}, \ell_{y}\right\rangle$, respectively, then we represent $x \oplus y$ by defining a tree $T$ as follows: we juxtapose $T_{x}$ and $T_{y}$ with a common root and take the union of the labelling functions. An example for $x=\mathrm{V}_{3} \oplus \mathrm{V}_{2} \oplus \mathrm{V}_{1}$ and $y=\left(\mathrm{V}_{4} \otimes \mathrm{V}_{2}\right) \oplus \mathrm{V}_{1}$ can be seen in Figure 1 .

(3) If $x, y \in \mathfrak{A}$ are represented by $\left\langle T_{x}, v_{x}\right\rangle$ and $\left\langle T_{y}, v_{y}\right\rangle$, respectively, then we represent $x \otimes y$ by defining a tree $T$ as follows: we start with $T_{y}$ and glue a copy of $T_{x}$ to each terminal node of $T_{x}$. An example for $x=\mathrm{V}_{3} \oplus \mathrm{V}_{2} \oplus \mathrm{V}_{1}$ and $y=\left(\mathrm{V}_{4} \otimes \mathrm{V}_{2}\right) \oplus \mathrm{V}_{1}$ can be seen in Figure 2 .

This corresponds directly to the representation of ordinal addition and multiplication by finite trees. Note that the order of the successors of a node in the tree is highly relevant, as ordinal addition and multiplication are not commutative. 


$$
T_{x} \quad T_{y} \quad T
$$

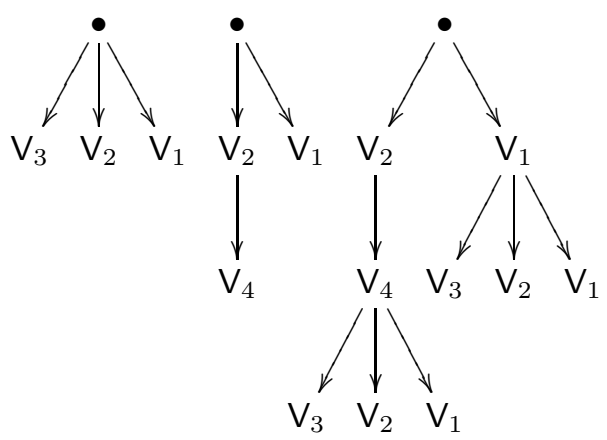

FiguRE 2. Multiplying the trees for $x=\mathrm{V}_{3} \oplus \mathrm{V}_{2} \oplus \mathrm{V}_{1}$ and $y=\left(\mathrm{V}_{4} \otimes \mathrm{V}_{2}\right) \oplus \mathrm{V}_{1}$.

We can now define the notion of a measure assignment. An order type is a function ot : $T \backslash\{\bullet\} \rightarrow$ Ord where $T$ is a finite tree with root $\bullet$. A germ is a function $\mathcal{G}$ defined on $T \backslash\{\bullet\}$ assigning a measure on some ordinal to each non-root node of the tree $T$. We say that a germ $\mathcal{G}$ lives on an order type ot if for each non-root node $v, \mathcal{G}(v)$ is a measure on ot $(v)$ and on no smaller ordinal. A pair of functions $\langle$ germ, ot $\rangle$ is a measure assignment on $\mathfrak{A}$ if ot and germ assign order types and germs to elements of $\mathfrak{A}$, respectively, such that for each $x \in \mathfrak{A}$, $\operatorname{germ}(x)$ lives on ot $(x)$.

Note that for a generator $\mathrm{v}$, the order type ot $(\mathrm{v})$ is essentially one ordinal, and the germ germ(v) is a measure on this ordinal. In the case of generators, we shall identify the order type and germ with the ordinal and measure, respectively.

It is clear that it is enough to specify the values of $\operatorname{germ}(v)$ and $\operatorname{ot}(v)$ for all $v \in \mathfrak{V}$ in order to fix the entire measure assignment: for an arbitrary term $x$ with labelled tree $\left\langle T_{x}, \ell_{x}\right\rangle$, each non-root node $v$ will be assigned the ordinal ot $(v):=\operatorname{ot}\left(\ell_{x}(v)\right)$ and the measure $\mu(v):=\operatorname{germ}\left(\ell_{x}(v)\right)$.

As our finite trees correspond to ordinal addition and multiplication, we can see $\operatorname{ot}(x)$ as a single ordinal computed recursively from the values of ot $(v)$ for the nodes $v$ of $T_{x}$. An example can be seen in Figure 3. We can, in fact, identify $\operatorname{ot}(x)$ with this ordinal; to be precise, we shall identify it with the presentation of the ordinal given by the tree. It is sometimes convenient to identify the domain of ot $(x)$ with the set of tuples $\left\langle i_{0}, \alpha_{0}, i_{1}, \alpha_{1}, \ldots, i_{k}, \alpha_{k}\right\rangle$ where $\left\langle i_{1}, \ldots, i_{k}\right\rangle$ is a terminal node of $T$, and each $\alpha_{\ell}<\operatorname{ot}(v)$ for $v=\left\langle i_{0}, \ldots, i_{\ell}\right\rangle$. The corresponding ordering is lexicographic ordering on these tuples. Clearly this ordering has order type ot $(x)$.

We say that the range of a measure assignment is the supremum of the ordinals $\operatorname{ot}(x)$ for all $x \in \mathfrak{A}$.

In a finite tree $T_{x}$, we call the rightmost immediate successor $v$ of the root the trailing node. If you consider the tree as an ordinal, then the term ot $(v)$ corresponds to the trailing term in the ordinal presentation of ot $(x)$. It will be important that $\operatorname{cf}(\operatorname{ot}(x))=\operatorname{cf}(\operatorname{ot}(v))$ for the trailing node $v$. 
(a)

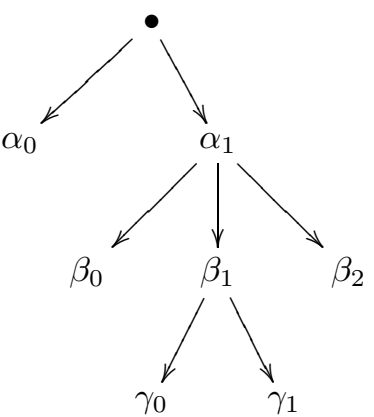

(b)

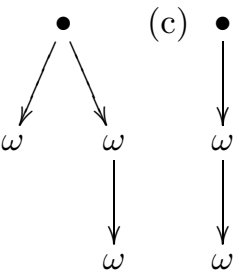

FiguRE 3. (a). The tree representation of $\alpha_{0}+\left(\beta_{0}+\left(\gamma_{0}+\gamma_{1}\right)\right.$. $\left.\beta_{1}+\beta_{2}\right) \cdot \alpha_{1}$. (b) \& (c). The (different) tree representations of $\omega+\omega^{2}=\omega^{2}$.

\section{Lifting AND CANONICITY}

It is our goal to analyse cardinals as ultrapowers via our measure assignments. In order to do this, we need to transform our germs into real measures on (odd) projective ordinals. We shall do this via lifting. We use two operations, the weak lift wlift $_{\kappa}$ and the strong lift slift $_{\kappa}$. The first uses the weak partition relation at $\kappa$ and the second the strong partition relation.

If $\kappa$ is an infinite cardinal and $T$ is a finite tree with a (finite) set $X$ of terminal nodes, there is a definable bijection $\ulcorner\urcorner:. \kappa^{X} \rightarrow \kappa$. We fix these bijections for the rest of the paper and use the same notation for all of these functions. As in the previous section, we fix an ordinal algebra $\mathfrak{A}$ with set of generator $\mathfrak{V}$. We also fix a measure assignment $\langle$ germ, ot $\rangle$ for $\mathfrak{A}$.

Fix a term $x \in \mathfrak{A}$ with labelled tree $\left\langle T_{x}, \ell_{x}\right\rangle$. For each terminal node $t=$ $\left\langle i_{0}, \ldots, i_{k}\right\rangle$ of $T_{x}$, consider the subset $\operatorname{ot}(x)_{t}$ of $\operatorname{ot}(x)$ consisting of those tuples $\left\langle j_{0}, \alpha_{0}, \ldots, j_{k}, \alpha_{k}\right\rangle$ with $\left\langle j_{0}, \ldots, j_{k}\right\rangle=\left\langle i_{0}, \ldots, i_{k}\right\rangle$. This set is naturally identified with $\operatorname{ot}\left(v_{i_{0}}\right) \times \operatorname{ot}\left(v_{i_{0}, i_{1}}\right) \times \cdots \times \operatorname{ot}\left(v_{i_{0}, \ldots, i_{k}}\right)$, where $v_{i_{0}, \ldots, i_{\ell}}=\ell_{x}\left(i_{0}, \ldots, i_{\ell}\right)$. We also have the product measure $\mu^{*}(t):=\mu\left(i_{0}\right) \times \mu\left(i_{0}, i_{1}\right) \times \cdots \times \mu\left(i_{0}, \ldots, i_{k}\right)$ on ot $_{t}(x)$, where $\mu\left(i_{0}, \ldots, i_{\ell}\right)$ is the measure assigned to the variable $v_{i_{0}, \ldots, i_{\ell}}$. Every function $f: \operatorname{ot}(x) \rightarrow$ Ord induces by restriction, for each terminal node $t$, a function $f^{t}: \operatorname{ot}(x)_{t} \rightarrow$ Ord. $f$ is, in a natural sense, the union of these subfunctions.

Notice that for each terminal node $t$ that is a successor of the trailing node of $T_{x}$, we have that $\sup \left(f^{t}\right)=\sup (f)$.

The term $x \in \mathfrak{A}$, along with our measure assignment, defines a germ $\mathcal{G}=$ $\operatorname{germ}(x)$. To every germ $\mathcal{G}$ and cardinal $\kappa$ having the weak partition relation we now associate a measure $\operatorname{wlift}_{\kappa}(\mathcal{G})$ as follows (we assume in the following definition that $\operatorname{ot}(x)<\kappa)$.

\section{Definition 6.}

wlift $_{\kappa}(\mathcal{G}):=\{A \subseteq \kappa$ : there is a club set $C \subseteq \kappa$ such that for all $f: \operatorname{ot}(\mathcal{G}) \rightarrow C$ of continuous type we have $\left.\left\ulcorner\left\{\left[f^{t}\right]_{\mu^{*}(t)}: t \in X\right\}\right\urcorner \in A\right\}$,

where $X$ is the set of terminal nodes of $T$. 
When the measure assignment is understood, we will simply write $\mathbf{w l i f t}_{\kappa}(x)$ for this measure.

The weak partition relation on $\kappa$ gives the following.

Theorem 7 (Weak Lifting Theorem). Let $\kappa$ be a weak partition cardinal closed under ultrapowers. Let $\mathcal{G}$ be a germ living on an order type $\operatorname{ot}(\mathcal{G})<\kappa$. Then $\operatorname{wlift}_{\kappa}(\mathcal{G})$ is a measure on $\kappa$.

Proof. Exercise. A full proof of the case of germs on trees of depth 1 can be found in [BoLö $\infty$, Theorem 10].

To see a few examples, we ask the reader to check that the lift of a principal ultrafilter on a singleton to any weak partition cardinal $\kappa$ is equal to $\mathcal{C}_{\kappa}^{\omega}$. Similarly, the lift of $\mathcal{C}_{\lambda}^{\omega}$ to $\kappa$ is $\mathcal{C}_{\kappa}^{\lambda}$.

The strong partition relation on $\kappa$ allows us to lift measures on $\kappa$ to measures on the ultrapower, according to the following definition.

Definition 8. Let $\kappa$ be a strong partition cardinal, $\mu$ a measure on $\kappa$, and $\lambda:=$ $\kappa^{\kappa} / \mu$. We define a measure on $\lambda$ by

$$
\begin{gathered}
\text { slift }_{\kappa}(\mu):=\{A \subseteq \lambda: \text { there is a club set } C \subseteq \kappa \text { such that for all } f: \kappa \rightarrow C \\
\text { of the correct type we have } \left.[f]_{\mu} \in A\right\} .
\end{gathered}
$$

We note that the measure $\mu$ determines $\kappa$, so we frequently just write $\operatorname{slift}(\mu)$. The strong partition relation at $\kappa$ gives immeduately the following.

Theorem 9 (Strong Lifting Theorem). Let $\kappa$ be a strong partition cardinal and $\mu$ be a measure on $\kappa$. Then $\operatorname{slift}(\mu)$ is a measure on $\kappa^{\kappa} / \mu$.

For notational convenience, we can combine the operations wlift and slift to a new operation that we call the high lift of $\mathcal{G}$ (here the germ $\mathcal{G}$ lives on $\varrho<\kappa$ and $\kappa$ is a strong partition cardinal closed under ultrapowers):

$$
\operatorname{highlift}_{\kappa}(\mathcal{G}):=\operatorname{slift}\left(\text { wlift }_{\kappa}(\mathcal{G})\right) .
$$

An important hypothesis for this paper is embodied in the following definition.

Definition 10. A measure assignment is called canonical for the strong partition cardinal $\kappa=\aleph_{\xi+1}$ if for all $x \in \mathfrak{A}$ with ot $(x)<\kappa$ we have

$$
\kappa^{\kappa} / \mathbf{w l i f t}_{\kappa}(x)=\aleph_{\xi+o(x)+1} .
$$

Note that canonicity implies a lot of non-obvious claims about the behaviour of sums and products of measures: while $o\left(\mathrm{~V}_{1} \oplus \mathrm{V}_{2}\right)=\omega=o\left(\mathrm{~V}_{2}\right)$, there is no a priori reason that the measures associated to these two terms should be similar. Canonicity of the measure assignment ensures that they are, in the sense that they give the same ultrapowers. Note that also implicit in canonicity is the claim that the bijection between $\kappa^{X}$ and $\kappa$ used in defining $\operatorname{wlift}_{\kappa}(x)$ does not effect the value of $\kappa^{\kappa} / \mathbf{w l i f t}_{\kappa}(x)$. To illustrate this last point let $\mathrm{W}_{1}^{3}$ be the three-fold product of the normal measure on $\omega_{1}$ (in the canonical measure assignment of the next section this will be wlift $\left.\omega_{\omega_{1}}\left(\mathrm{~V}_{1} \oplus \mathrm{V}_{1} \oplus \mathrm{V}_{1}\right)\right)$. Let $\mu_{1}$ be the measure on $\omega_{1}$ obtained from $\mathrm{W}_{1}^{3}$ by identifying $\left(\omega_{1}\right)^{3}$ with $\omega_{1}$ using the ordering $\left\langle\alpha_{1}, \alpha_{2}, \alpha_{3}\right\rangle<_{1}\left\langle\beta_{1}, \beta_{2}, \beta_{3}\right\rangle$ iff $\left\langle\alpha_{3}, \alpha_{1}, \alpha_{2}\right\rangle<_{\text {lex }}\left\langle\beta_{3}, \beta_{1}, \beta_{2}\right\rangle$, where $<_{\text {lex }}$ denotes lexicographic ordering. Let $\mu_{2}$ be defined similarly but using $\left\langle\alpha_{1}, \alpha_{2}, \alpha_{3}\right\rangle<_{2}\left\langle\beta_{1}, \beta_{2}, \beta_{3}\right\rangle$ 
iff $\left\langle\alpha_{3}, \alpha_{2}, \alpha_{1}\right\rangle<_{\operatorname{lex}}\left\langle\beta_{3}, \beta_{2}, \beta_{1}\right\rangle$. Let $\nu_{1}=\operatorname{slift}\left(\mu_{1}\right)$ and $\nu_{2}=\operatorname{slift}\left(\mu_{2}\right)$. Then $\nu_{1}$, $\nu_{2}$ are both measures on $\omega_{4}$, but are quite different and definitely non-equivalent measures. Nevertheless, $\boldsymbol{\delta}_{3}^{1} \boldsymbol{\delta}_{3}^{1} / \nu_{1}=\delta_{3}^{1_{3}^{1}} / \nu_{2}$. In fact, the description analysis readily shows that both ultrapowers are equal to $\aleph_{\omega^{\omega^{3}+1}}$, which will also follow from our canonicity assumption (the measure assignment of the next section will assign to $\mathrm{V}_{5}$ the measure highlift $\omega_{\omega_{1}}\left(\mathrm{~V}_{1} \oplus \mathrm{V}_{1} \oplus \mathrm{V}_{1}\right)$, which is $\nu_{1}$ or $\nu_{2}$ depending on the bijection used, and $\left.o\left(\mathrm{~V}_{5}\right)=\omega^{\omega^{3}}\right)$.

\section{A ReCuRsive Definition of A MEASURE ASSignment}

In this section, we shall define a measure assignment for the algebra $\mathfrak{A}_{\varepsilon_{0}}$. Recall that $\mathbf{e}_{0}:=0$ and $\mathbf{e}_{n+1}:=\omega^{\left(\omega^{\mathbf{e}_{n}}\right)}$; with this notation, we have that $\mathfrak{A}_{\varepsilon_{0}}=\bigcup_{n \in \omega} \mathfrak{A}_{\mathbf{e}_{n}}$. The assignment will be defined recursively along this union of algebras. ${ }^{1}$ The basic idea is that the variables at a given level correspond to the high lifts of the terms (not just the variables) at the previous levels.

A standing assumption for the rest of this paper is that all the odd projective ordinals $\boldsymbol{\delta}_{2 n+1}^{1}$ have the strong partition property and are closed under ultrapowers. The reader may, if desired, add this to our canonicity assumption.

We start with $\mathfrak{A}_{2}$ generated by $\bigvee_{0}$ and $\bigvee_{1}$. We have to deal with an anomaly at the beginning: we want to set $\operatorname{ot}\left(\mathrm{V}_{0}\right):=0$, but of course there is no measure on the empty set. For the purpose of this definition, we declare $\varnothing$ to be a measure on 0 , and we define wlift $_{\kappa}(\varnothing)$ to be any principal ultrafilter on $\kappa=\aleph_{\xi+1}$. Clearly, this fits well, as $o\left(\mathrm{~V}_{0}\right)=0$ and thus

$$
\kappa^{\kappa} / \text { wlift }_{\kappa}\left(\mathrm{V}_{0}\right)=\aleph_{\xi+o}\left(\mathrm{~V}_{0}\right)+1=\aleph_{\xi+1}=\kappa .
$$

We continue our definition by setting $\operatorname{ot}\left(\mathrm{V}_{1}\right):=1$ and $\operatorname{germ}\left(\mathrm{V}_{1}\right)$ to be the principal ultrafilter on 1. Lifting $\operatorname{germ}\left(\bigvee_{1}\right)$ to $\boldsymbol{\delta}_{1}^{1}=\aleph_{1}$, we get that $\operatorname{wlift}_{\boldsymbol{\delta}_{1}^{1}}\left(\bigvee_{1}\right)$ is the club filter on $\omega_{1}$. Again, this conforms with the canonicity requirement, as

$$
\aleph_{2}=\aleph_{1}^{\aleph_{1}} / \mathcal{C}_{\omega_{1}}=\aleph_{1}^{\aleph_{1}} / \text { wlift }_{\delta_{1}^{1}}\left(\mathrm{~V}_{1}\right)=\aleph_{0+o\left(\mathrm{~V}_{1}\right)+1}
$$

We shall now lift the measure assignment from $\mathfrak{A}_{2}$ to $\mathfrak{A}_{\omega}$, or more generally, from $\mathfrak{A}_{2+\mathbf{e}_{n}}$ to $\mathfrak{A}_{\mathrm{ht}\left(\mathfrak{A}_{2+\mathbf{e}_{n}}\right)}=\mathfrak{A}_{\mathbf{e}_{n+1}}$.

Suppose that we have a measure assignment for $\mathfrak{A}_{2+\mathbf{e}_{n}}$ with range $<\boldsymbol{\delta}_{2 n+1}^{1}$. For $\xi<2+\mathbf{e}_{n}$, we leave $\operatorname{ot}\left(\mathrm{V}_{\xi}\right)$ and $\operatorname{germ}\left(\mathrm{V}_{\xi}\right)$ unchanged. If $\xi=2+\mathbf{e}_{n}+\eta$, where $\eta<\mathbf{e}_{n+1}$, then there is some term $y \in \mathfrak{A}_{2+\mathbf{e}_{n}}$ such that $\eta=o(y)$. We use the Cantor normal form of $\eta$ to get a canonical representative $y$. We now define

$$
\begin{gathered}
\operatorname{ot}\left(\mathrm{V}_{\xi}\right):=\boldsymbol{\delta}_{2 n+1}^{1} \boldsymbol{\delta}_{2 n+1}^{1} / \text { wlift }_{\boldsymbol{\delta}_{2 n+1}^{1}}(y), \text { and } \\
\operatorname{germ}\left(\mathrm{V}_{\xi}\right):=\operatorname{highlift}_{\boldsymbol{\delta}_{2 n+1}^{1}}(\operatorname{germ}(y))
\end{gathered}
$$

Note that in order to define $\operatorname{germ}\left(\mathrm{V}_{\xi}\right)$ for $\mathbf{e}_{n} \leq \xi<\mathbf{e}_{n+1}$ we need to know that wlift $_{\boldsymbol{\delta}_{2 n+1}^{1}}(y)$ is a measure on $\boldsymbol{\delta}_{2 n+1}^{1}$, which follows from the closure of $\boldsymbol{\delta}_{2 n+1}^{1}$ under ultrapowers. From the closure of $\boldsymbol{\delta}_{2 n+3}^{1}$ under ultrapowers it then follows that ot $\left(\mathrm{V}_{\xi}\right)<\boldsymbol{\delta}_{2 n+3}^{1}$.

This finishes the definition of a measure assignment for $\mathfrak{A}_{\varepsilon_{0}}$. Again, we invite the reader to compare the recursive definition with the table in Figure 5: the first

\footnotetext{
${ }^{1}$ It may be convenient for the reader to accompany the reading of the recursive definition with the table in Figure 5 which gives all of the relevant values for terms in $\mathfrak{A}_{\omega^{\omega}} \omega$.
} 
system of the table gives the values in $\mathfrak{A}_{2}$ (before the vertical line) and $\mathfrak{A}_{\omega}$ (after the vertical line) and the second system gives the values in $\mathfrak{A}_{\mathbf{e}_{2}}$ with the columns in the second system corresponding to the columns in the first system via the high lift.

To get acquainted with this definition, let us compute the values of $\operatorname{germ}\left(\mathrm{V}_{n}\right)$ for $n<\omega$.

- We have $\mathrm{V}_{2}=\mathrm{V}_{2+\mathbf{e}_{0}+0}$, so the canonical representative $y$ of $\eta=0$ will be just $\mathrm{V}_{0}$. Then wlift $_{\boldsymbol{\delta}_{1}^{1}}\left(\mathrm{~V}_{0}\right)$ is just the principal ultrafilter by convention. Thus, $\operatorname{germ}\left(\mathrm{V}_{2}\right)$ is the strong lift of the principal ultrafilter which is the normal measure $\mathrm{W}_{1}^{1}$ on $\omega_{1}$.

- Now, we have $\mathrm{V}_{3}=\mathrm{V}_{2+\mathbf{e}_{0}+1}$, so $\eta=1$ and thus our canonical $y$ is $\mathrm{V}_{1}$. Lifting the principal filter on 1 to $\omega_{1}$ yields the normal measure $\mathrm{W}_{1}^{1}$ on $\omega_{1}$ as $\mathrm{W}_{1}^{1}=$ wlift $_{\boldsymbol{\delta}_{1}^{1}}\left(\mathrm{~V}_{1}\right)$. As we know, $\aleph_{1} \aleph_{1} / \mathrm{W}_{1}^{1}=\aleph_{2}$, $\operatorname{so} \operatorname{ot}\left(\mathrm{V}_{3}\right)=\omega_{2}$. So, $\operatorname{germ}\left(\mathrm{V}_{2}\right)=\operatorname{slift}\left(\mathrm{W}_{1}^{1}\right)=$ the $\omega$-club filter on $\omega_{2}$ (this is denoted $S_{1}^{1}$ in [Ja99, Definition 1.3]).

- For $n \geq 3, \mathrm{~V}_{n}=\mathrm{V}_{2+\mathbf{e}_{0}+(n-2)}$, so $\eta=n-2$, and our term is $y=\mathrm{V}_{1} \otimes(n-2)$. Also, wlift $\boldsymbol{\delta}_{1}^{1}(y)=\mathrm{W}_{1}^{n-2}$, the $(n-2)$-fold product of the normal measure on $\omega_{1}$. If we identify $\left(\omega_{1}\right)^{n-2}$ with $\omega_{1}$ via the ordering $\left(\alpha_{1}, \ldots, \alpha_{n-2}\right)<$ $\left(\beta_{1}, \ldots, \beta_{n-2}\right)$ iff $\left(\alpha_{n-2}, \alpha_{1}, \ldots, \alpha_{n-3}\right)<_{\operatorname{lex}}\left(\beta_{n-2}, \beta_{1}, \ldots, \beta_{n-3}\right)$, then the resulting measure on $\omega_{n-1}$ is denoted $\mathrm{S}_{1}^{n-2}$ in [Ja99, Definition 1.3]. So, using this bijection, $\operatorname{germ}\left(\mathrm{V}_{n}\right)=\mathrm{S}_{1}^{n-2}$.

Computing the ultrapowers of $\boldsymbol{\delta}_{3}^{1}$ with the measures associated to $V_{2}$ and $V_{3}$ gives exactly the right answers:

$$
\begin{gathered}
\boldsymbol{\delta}_{3}^{1} \boldsymbol{\delta}_{3}^{1} / \text { wlift }_{\boldsymbol{\delta}_{3}^{1}}\left(\mathrm{~V}_{2}\right)=\boldsymbol{\delta}_{3}^{1} \boldsymbol{\delta}_{3}^{1} / \mathcal{C}_{\boldsymbol{\delta}_{3}^{1}}^{\omega_{1}}=\aleph_{\omega \cdot 2+1}=\aleph_{\omega+o\left(\mathrm{~V}_{2}\right)+1}, \text { and } \\
\boldsymbol{\delta}_{3}^{1} \boldsymbol{\delta}_{3}^{1} / \text { wlift }_{\boldsymbol{\delta}_{3}^{1}}\left(\bigvee_{3}\right)=\boldsymbol{\delta}_{3}^{1} \boldsymbol{\delta}_{3}^{1} / \mathcal{C}_{\boldsymbol{\delta}_{3}^{1}}^{\omega_{2}}=\aleph_{\omega^{\omega}+1}=\aleph_{\omega+o\left(\mathrm{~V}_{3}\right)+1} .
\end{gathered}
$$

Rephrased in the language of measure assignments, we can interpret Kleinberg's Theorem 3 and the results from [JaKh $\infty$ ] as follows:

Theorem 11 (Kleinberg). The given measure assignment on $\mathfrak{A}_{2}$ is canonical for $\boldsymbol{\delta}_{1}^{1}$.

Theorem 12 (Jackson-Khafizov). The given measure assignment on $\mathfrak{A}_{\omega}=\mathfrak{A}_{\mathbf{e}_{1}}$ is canonical for $\boldsymbol{\delta}_{3}^{1}=\boldsymbol{\delta}_{2 \cdot 1+1}^{1}$.

The upper and lower bound computations underlying [Ja88, Ja99, JaKh $\infty$ ] indicate strongly that canonicity will hold everywhere. We call the assumption that the measure assignment defined above on $\mathfrak{A}_{\mathbf{e}_{n}}$ is canonical for $\boldsymbol{\delta}_{2 n+1}^{1}$ the canonicity assumption.

\section{Applications of the Canonicity Assumption}

In this section we shall work under the canonicity assumption. Based on that assumption, we shall be able to give algorithms to compute the cofinalities of all cardinals below $\aleph_{\varepsilon_{0}}$ and the Kleinberg sequences derived from the normal ultrafilters on the odd projective ordinals. 
6.1. Computation of regular cardinals. As a first step in the computation of the regular cardinals, we shall give an algorithm that identifies special variables in the set $\mathfrak{V}$ of generators. We call these variables normal, as they will be the ones that are assigned normal measures by our recursive assignment.

We say that $V_{0}$ and $V_{1}$ are normal. In each of the iteration steps from $\mathfrak{A}_{2+\mathbf{e}_{n}}$ to $\mathfrak{A}_{\mathbf{e}_{n+1}}$, we identify the folowing new variables as normal: for $\xi=2+\mathbf{e}_{n}+\eta$ for some $\eta<\mathbf{e}_{n+1}$, the variable $\mathrm{V}_{\xi}$ is normal if and only if $\eta=o(\mathrm{v})$ for some normal $\vee \in \mathfrak{A}_{2+\mathbf{e}_{n}}$.

By Proposition 5, for infinite ordinals $\xi$, the function $o$ is just $\xi \mapsto \omega^{\omega^{\xi}}$, therefore, we can easily compute the indices of the normal variables by the following algorithm: write down the $2^{n+1}$ normal variables for $\mathfrak{A}_{\mathbf{e}_{n}}$, write down the values of $o$ for these variables underneath the variables, then compute the indices of the $2^{n+1}$ new normal variables as $\mathbf{e}_{n+1}+o(\mathrm{v})$ for the values of $o$ in your list. You can see the first three steps of the algorithm in the following table:

\begin{tabular}{|c|c|c|c|}
\hline $\mathfrak{A}_{2}$ & $\begin{array}{l}0 \\
0\end{array}$ & $\begin{array}{l}1 \\
1\end{array}$ & \\
\hline $\mathfrak{A}_{\omega}$ & $\begin{array}{c}2=2+0 \\
\omega\end{array}$ & $\begin{array}{c}3=2+1 \\
\omega^{\omega}\end{array}$ & \\
\hline $\mathfrak{A}_{\mathbf{e}_{2}}$ & $\begin{array}{l}\omega=\omega+0 \\
\mathbf{e}_{2}=\omega^{\omega}\end{array}$ & $\begin{array}{l}\omega+1 \\
\omega^{\omega^{\omega+1}}\end{array}$ & $\begin{array}{cc}\omega \cdot 2=\omega+\omega & \omega^{\omega}=\omega+\omega^{\omega} \\
\omega^{\omega^{\omega \cdot 2}} & \omega^{\omega^{\omega}}\end{array}$ \\
\hline
\end{tabular}

We shall prove inductively in a series of lemmas that the normal variables give rise to normal measures, first lifting a normal measure on $\varrho<\kappa$ to $\mathcal{C}_{\kappa}^{\varrho}$ by the operation wlift (Lemma 13) and then lifting the (semi-)normal measure on $\kappa$ to a normal measure by the operation slift (Lemma 15):

Lemma 13. Let $\kappa$ be a strong partition cardinal closed under ultrapowers. If $\mu$ is a normal measure on $\varrho<\kappa$, then wlift $_{\kappa}(\mu)$ is a normal measure.

Proof. Recall that wlift $_{\kappa}(\mu)$ is the measure on $\kappa$ defined by: $A$ has measure one if and only if there is a club set $C \subseteq \kappa$ such that for all $f: \varrho \rightarrow C$ of continuous type, we have that $[f]_{\mu} \in A$.

For any $f$ of this type, if $\sup (f)$ is closed under ultrapowers then $[f]_{\mu}=\sup (f)$ by normality of $\mu$. Also, for any club set $C \subseteq \kappa$ and any limit point $\alpha$ of $C$ of cofinality $\varrho$, there is an $f: \varrho \rightarrow C$ of this type with $\sup (f)=\alpha$.

Thus, $A$ has measure one if and only if there is a club set $C \subseteq \kappa$ such that all $\alpha \in C$ of cofinality $\varrho$ are in $A$. It is well-known that the weak partition relation on $\kappa$ implies that this describes a normal measure, that is, $\operatorname{wlift}_{\kappa}(\mu)$ is the $\varrho$-cofinal normal measure on $\kappa$.

Lemma 14. Let $\nu$ be any semi-normal measure on the strong partition cardinal $\kappa$ (i.e., one that contains all club subsets of $\kappa$ ). If $f, g: \kappa \rightarrow \kappa$ are of the correct type with $[f]_{\nu}<[g]_{\nu}$, then there are $f^{\prime}, g^{\prime}$ of the correct type with $\left[f^{\prime}\right]_{\nu}=[f]_{\nu},\left[g^{\prime}\right]_{\nu}=$ $[g]_{\nu}$, and $f^{\prime}(\alpha)<g^{\prime}(\alpha)<f^{\prime}(\alpha+1)$ for all $\alpha<\kappa$. Furthermore, $\operatorname{ran}\left(f^{\prime}\right) \subseteq \operatorname{ran}(f)$ and $\operatorname{ran}\left(g^{\prime}\right) \subseteq \operatorname{ran}(g)$.

Proof. Define $f^{\prime}, g^{\prime}$ recursively by letting $f^{\prime}(\alpha)$ be the least element in the range of $f$ greater than $\sup _{\beta<\alpha} g^{\prime}(\beta)$. Let $g^{\prime}(\alpha)$ be the least element in the range of $g$ which is greater than $f^{\prime}(\alpha)$. Clearly there is a club set $C \subseteq \kappa$ (the points closed under $g^{\prime}$ ) on which $f^{\prime}=f$. Since $\nu$ is semi-normal, $\left[f^{\prime}\right]_{\nu}=[f]_{\nu}$. If $A$ is the $\nu$ measure 
one set on which $g(\alpha)>f(\alpha)$, then for $\alpha \in C \cap A$ we have $g^{\prime}(\alpha)=g(\alpha)$. Thus, $[g]_{\nu}=\left[g^{\prime}\right]_{\nu}$ by semi-normality.

Lemma 15. Let $\nu$ be any semi-normal measure on the strong partition cardinal $\kappa$. Then $\mu:=\operatorname{slift}(\nu)$ is a normal measure.

Proof. Let $\vartheta=\kappa^{\kappa} / \nu$, so $\mu$ is a measure on $\vartheta$. Fix $F: \vartheta \rightarrow \vartheta$ which is pressing down.

Consider first the partition $\mathcal{P}_{1}$ where we partition pairs of functions $\langle f, g\rangle$ where $f, g: \kappa \rightarrow \kappa$ are of the correct type and $f(\alpha)<g(\alpha)<f(\alpha+1)$ for all $\alpha<\kappa$ according to whether $[f]_{\nu}>F\left([g]_{\nu}\right)$. We claim that on the homogeneous side thew stated property holds. Towards a contradiction, suppose $C$ is club and homogeneous for the contrary side.

Fix $g: \kappa \rightarrow C^{\prime}$ of the correct type, where $C^{\prime}$ is the set of closure points of $C$ (i.e., the $\alpha \in C$ such that $\alpha$ is the $\alpha$ th element of $C$ ). Since $F\left([g]_{\nu}\right)<[g]_{\nu}$, we may get $f: \kappa \rightarrow C$ with $F\left([g]_{\nu}\right)<[f]_{\nu}<[g]_{\nu}$. Let $f^{\prime}, g^{\prime}$ be obtained from Lemma 14. Then $f^{\prime}, g^{\prime}$ are of the correct type, ordered as in $\mathcal{P}_{1}$, and have range in $C$, but $\left[f^{\prime}\right]_{\nu}=[f]_{\nu}>F\left([g]_{\nu}\right)=F\left(\left[g^{\prime}\right]_{\nu}\right)$, a contradiction to the definition of $C$. Let now $C_{1}$ be club and homogeneous for the stated side of the partition. Fix $f: \kappa \rightarrow C_{1}$ of the correct type and let $\delta=[f]_{\nu}$.

Then for any $g: \kappa \rightarrow C_{1}$ of the correct type with $[g]_{\nu}>\delta$ we have $F\left([g]_{\nu}\right)<\delta$. This follows from the definition of $C_{1}$ and Lemma 14. This shows $\mu$ is weakly normal, that is, any pressing down function is bounded on a measure one set.

Consider next the partition $\mathcal{P}_{2}$ where we partition pairs $\langle f, g\rangle$ of the same type as in $\mathcal{P}_{1}$ but now partitioned according to whether $F\left([f]_{\nu}\right) \leq F\left([g]_{\nu}\right)$. We claim that on the homogeneous side the stated property holds. Suppose not and let $C$ be homogeneous for the contrary side.

We can easily construct functions $f_{i}: \kappa \rightarrow C$ of the correct type such that $f_{i}(\alpha)<f_{i+1}(\alpha)$ and $f_{i}(\alpha)<f_{0}(\alpha+1)$ for all $i \in \omega$ and $\alpha<\kappa$. But then $F\left(\left[f_{0}\right]_{\nu}\right)>F\left(\left[f_{1}\right]_{\nu}\right)>\ldots$, a contradiction. Fix a club set $C_{2} \subseteq \kappa$ homogeneous for the stated side of $\mathcal{P}_{2}$.

Consider a third partition $\mathcal{P}_{3}$ where we partition pairs $\langle f, g\rangle$ of the same type again according to whether $F\left([f]_{\nu}\right)=F\left([g]_{\nu}\right)$. If there is a club set $C \subseteq \kappa$ homogeneous for the stated side of $\mathcal{P}_{3}$, then we are done since Lemma 14 implies that for any $f, g: \kappa \rightarrow C$ of the correct type we have $F\left([f]_{\nu}\right)=F\left([g]_{\nu}\right)$.

Suppose $C_{3}$ is homogeneous for the contrary side of $\mathcal{P}_{3}$. Let $C=C_{1} \cap C_{2} \cap C_{3}$. Fix $f: \kappa \rightarrow \kappa$ with $[f]_{\nu}>\delta$. Let $h:\{(\alpha, \beta): \alpha<f(\beta)\} \rightarrow C$ be of uniform cofinality $\omega$, discontinuous, and order-preserving with respect to reverse lexicographic ordering. Define a map $\pi:[f]_{\nu} \rightarrow \delta$ as follows. Let $\gamma=[g]_{\nu}<[f]_{\nu}$. Let $\pi(\gamma)=\left[g^{\prime}\right]_{\nu}$, where $g^{\prime}(\beta)=h(g(\beta), \beta)$ if $g(\beta)<f(\beta)$, and $=h(0, \beta)$ otherwise. It is now easy to check that $\pi$ is a well-defined, order-preserving map from $[f]_{\nu}$ into $\delta$, a contradiction since $[f]_{\nu}>\delta$.

Theorem 16. The measure assignment from $\oint 5$ assigns normal measures to all normal variables. Consequently, $\boldsymbol{\delta}_{2 n+1}^{1} \boldsymbol{\delta}_{2 n+1}^{1} / \mathbf{w l i f t}_{\boldsymbol{\delta}_{2 n+1}^{1}}(\mathrm{v})$ is a regular cardinals for all normal variables $v$.

Proof. The first part of the claim follows immediately by induction from Lemmas 13 and 15. The second part follows from the fact that if there is a normal measure on $\lambda$, then $\lambda$ must be regular. 


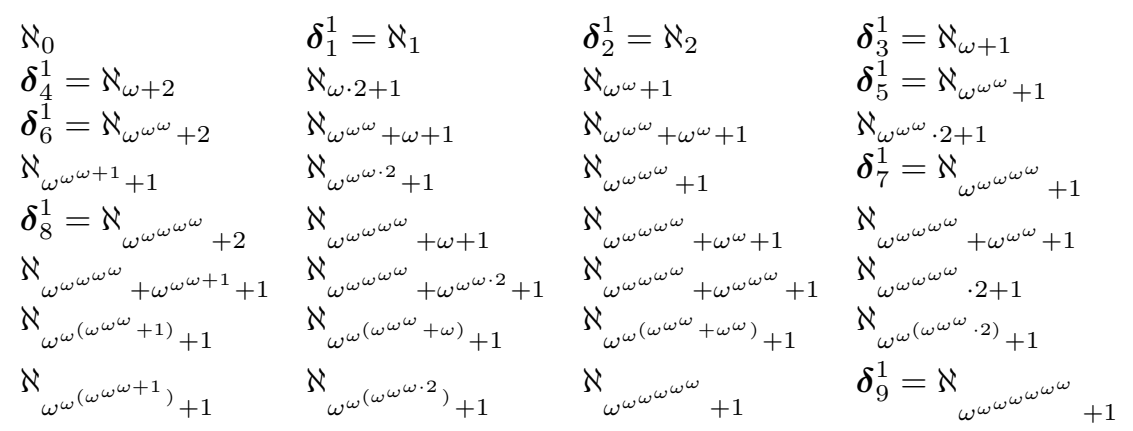

FiguRE 4 . The first 32 regular cardinals.

Assuming that the measure assignment is canonical, we can now compute these regular cardinals easily from the table given above by looking at the row containing the $o$-values.

In the table in Figure 4, we list the first 32 of such cardinals (up to $\boldsymbol{\delta}_{9}^{1}$ ). Note that at the point we have not yet proved that these are all the regular cardinals. This will follow from the next algorithm.

6.2. Computation of the Cofinalities. In $\S 6.1$, we singled out special variables in our algebra and proved in Theorem 16 that each of these gives rise to a regular ultrapower. In this section, we shall now reduce the computation of all cofinalities to the cofinalities associated to the normal variables. This argument will be done inductively based on the following elementary yet powerful result:

Lemma 17. Let $\mu$ be a measure on $\varrho$ with $\operatorname{cf}(\varrho)=\delta$. Let $\kappa$ be a weak partition cardinal closed under ultrapowers such that $\varrho<\kappa$. Then there is a cofinal embedding from $\kappa^{\kappa} / \mathcal{C}_{\kappa}^{\delta}$ into $\kappa^{\kappa} / \mathbf{w l i f t}_{\kappa}(\mu)$.

Proof. For $F: \kappa \rightarrow \kappa$, let $\pi(F)=G$ be defined as follows: for $\alpha<\kappa$ represented by $g: \varrho \rightarrow \kappa$ of continuous type, let $G\left([g]_{\mu}\right)=F(\sup (g))$. This is well-defined since if $\left[g_{1}\right]_{\mu}=\left[g_{2}\right]_{\mu}$ and $g_{1}, g_{2}$ are both increasing, then $\sup \left(g_{1}\right)=\sup \left(g_{2}\right)$ (since any $\mu$ measure one set is cofinal in $\varrho$ ).

Suppose $\left[F_{1}\right]_{\mathcal{C}_{\kappa}^{\delta}}=\left[F_{2}\right]_{\mathcal{C}_{\kappa}^{\delta}}$. Let $C \subseteq \kappa$ be a club set such that for all $\alpha \in C$ of cofinality $\delta$ we have $F_{1}(\alpha)=F_{2}(\alpha)$. Then for any $\beta<\kappa$ represented by a $g: \varrho \rightarrow C$ of continuous type we have $G_{1}(\beta)=F_{1}(\sup (g))=F_{2}(\sup (g))=G_{2}(\beta)$ whence $\left[G_{1}\right]_{\text {wlift }_{\kappa}(\mu)}=\left[G_{2}\right]_{\text {wlift }_{\kappa}(\mu)}$. Thus, $\pi$ gives a well-defined map from $\kappa^{\kappa} / \mathcal{C}_{\kappa}^{\delta}$ into $\kappa^{\kappa} /$ wlift $_{\kappa}(\mu)$.

To see this is cofinal, let $G: \kappa \rightarrow \kappa$. For $\alpha<\kappa$ of cofinality $\delta$, define $F(\alpha)=$ $\sup \left\{G\left([g]_{\mu}\right) ; \sup (g)=\alpha\right\}$, where the supremum ranges over $g$ of continuous type. This is well-defined as $\kappa$ is regular and closed under ultrapowers.

Then $\pi\left([F]_{\mathcal{C}_{\kappa}^{\delta}}\right)>[G]_{\text {wlift }_{\kappa}(\mu)}$ since for all $g: \varrho \rightarrow \kappa$ of continuous type we have $\pi(F)\left([g]_{\mu}\right)=F(\sup (g))=\sup \left\{G\left(\left[g^{\prime}\right]_{\mu}\right): \sup \left(g^{\prime}\right)=\sup (g)\right\} \geq G\left([g]_{\mu}\right)$.

As an immediate consequence, we can reduce the computation of the cofinality of $\kappa^{\kappa} /$ wlift $_{\kappa}(x)$ for an arbitrary term $x \in \mathfrak{A}_{\varepsilon_{0}}$ to the cofinalities of the basic variables:

Corollary 18. Let $x \in \mathfrak{A}_{\varepsilon_{0}}$ be a term with trailing node $v$ such that $\ell_{x}(v)=\mathrm{v}$. If $o(x)<\mathbf{e}_{n+1}$, write $\kappa:=\boldsymbol{\delta}_{2 n+1}^{1}$ and $\lambda:=\kappa^{\kappa} /$ wlift $_{\kappa}(\mathrm{v})$. Then

$$
\operatorname{cf}\left(\kappa^{\kappa} / \mathbf{w l i f t}_{\delta_{2 n+1}^{1}}(x)\right)=\operatorname{cf}(\lambda) .
$$


Proof. This is immediate from Lemma 17 keeping in mind that $\operatorname{cf}(\operatorname{ot}(x))=\operatorname{cf}(\operatorname{ot}(v))$.

We shall now recursively define a function nor: $\mathfrak{V} \rightarrow \mathfrak{V}$ assigning normal variables to arbitrary generators in the algebra. Our recursion will go along the tower of algebras $\mathfrak{A}_{2+\mathbf{e}_{n}}$ as the definition of the measure assignment in $\S 5$.

In $\mathfrak{A}_{2}$, all basic variables are normal, so the function nor can just be the identity. Suppose that we have defined the function nor on $\mathfrak{A}_{2+\mathbf{e}_{n}}$ and want to extend it to $\mathfrak{A}_{\mathbf{e}_{n+1}}$. Each of the generators $\bigvee_{\alpha}$ of $\mathfrak{A}_{\mathbf{e}_{n+1}}$ was either already in $\mathfrak{A}_{2+\mathbf{e}_{n}}$ or is of the form $\mathrm{V}_{2+\mathbf{e}_{n}+\xi}$ for some $\xi<\mathrm{ht}\left(\mathfrak{A}_{2+\mathbf{e}_{n}}\right)$. By the recursive measure assignment from $\S 5$, this variable $\mathrm{V}_{\alpha}=\mathrm{V}_{2+\mathbf{e}_{n}+\xi}$ is linked to terms $x \in \mathfrak{A}_{2+\mathbf{e}_{n}}$ such that $o(x)=\xi$. Let $x$ be such a term with representing tree $\left\langle T_{x}, \ell_{x}\right\rangle$ and trailing node $v \in T_{x}$. Then $\ell_{x}(v)$ is a generator of $\mathfrak{A}_{2+\mathbf{e}_{n}}$.

We can now define

$$
\operatorname{nor}\left(\mathrm{V}_{\alpha}\right):=\mathrm{V}_{2+\mathbf{e}_{n}+o\left(\operatorname{nor}\left(\ell_{x}(v)\right)\right)} .
$$

Theorem 19. For each generator $\mathrm{v}$ of $\mathfrak{A}_{\varepsilon_{0}}$ and every odd projective ordinal $\kappa=$ $\boldsymbol{\delta}_{2 n+1}^{1}$ such that ot $(\mathrm{v})<\kappa$, we have that

$$
\operatorname{cf}\left(\kappa^{\kappa} / \mathbf{w l i f t}_{\kappa}(\mathrm{v})\right)=\operatorname{cf}\left(\kappa^{\kappa} / \mathbf{w l i f t}_{\kappa}(\operatorname{nor}(\mathrm{v}))\right)
$$

Proof. The claim is proved by induction on $n$. Recall that the generators $v$ with $\operatorname{ot}(\mathbf{v})<\boldsymbol{\delta}_{2 n+1}^{1}$ are precisely those in $\mathfrak{A}_{2+\mathbf{e}_{n}}$. The case $n=0$ is trivial as nor is the identity on the generators in $\mathfrak{A}_{2}$ (i.e., $\mathrm{V}_{0}, \mathrm{~V}_{1}$ ). Assume the theorem holds for $n$, that is for $\boldsymbol{\delta}_{2 n+1}^{1}$ and $\mathfrak{A}_{2+\mathbf{e}_{n}}$, and we show it holds for $n+1$, that is, for $\boldsymbol{\delta}_{2 n+3}^{1}$ and $\mathfrak{A}_{\mathbf{e}_{n+1}}$.

Let $\mathrm{v}$ be a generator in $\mathfrak{A}_{\mathbf{e}_{n+1}}$, so $\mathbf{v}=\mathrm{V}_{2+\mathbf{e}_{n}+\xi}$ for some $\xi<\mathbf{e}_{n+1}=\operatorname{ht}\left(\mathfrak{A}_{2+\mathbf{e}_{n}}\right)$. Fix $x \in \mathfrak{A}_{2+\mathbf{e}_{n}}$ such that $o(x)=\xi$, let $v$ be the trailing term of $\left\langle T_{x}, \ell_{x}\right\rangle$, and $\mathrm{v}^{*}:=\ell_{x}(v)$. By definition of nor, we have nor $(\mathrm{v})=\mathrm{V}_{2+\mathbf{e}_{n}+o\left(\operatorname{nor}\left(\mathrm{v}^{*}\right)\right)}$. Let $\lambda:=\boldsymbol{\delta}_{2 n+1}^{1}$. By Corollary 18 and the induction hypothesis, we have that

$$
\operatorname{cf}\left(\lambda^{\lambda} / \mathbf{w l i f t}_{\lambda}(x)\right)=\operatorname{cf}\left(\lambda^{\lambda} / \mathbf{w l i f t}_{\lambda}\left(\mathrm{v}^{*}\right)\right) .
$$

But $\lambda^{\lambda} /$ wlift $_{\lambda}(x)=\operatorname{ot}(\mathrm{v})$ and $\lambda^{\lambda} / \mathbf{w l i f t}_{\lambda}\left(\mathrm{v}^{*}\right)=\operatorname{ot}(\operatorname{nor}(\mathrm{v}))$. Now we can apply Lemma 17 (with the $\kappa$ there being $\boldsymbol{\delta}_{2 n+3}^{1}$ ) to finish the claim.

Using Corollary 18 and Theorem 19, we can now describe the algorithm to compute the value of $\operatorname{cof}_{\kappa}(x):=\operatorname{cf}\left(\kappa^{\kappa} /\right.$ wlift $\left._{\kappa}(x)\right)$ recursively for arbitrary $x$. Suppose that we have already computed $\operatorname{cof}_{\kappa} \mid \mathfrak{A}_{2+\mathbf{e}_{n}}$ for all odd projective ordinals $\kappa \geq \boldsymbol{\delta}_{2 n+1}^{1}$. We shall give an algorithm to compute cof ${ }_{\kappa} \mid \mathfrak{A}_{\mathbf{e}_{n+1}}$ for all $\kappa \geq \boldsymbol{\delta}_{2 n+3}^{1}$.

\section{Algorithm.}

Given a term $x \in \mathfrak{A}_{\mathbf{e}_{n+1}}$, ask whether $x \in \mathfrak{A}_{2+\mathbf{e}_{n}}$ or not.

Case 1. $x \in \mathfrak{A}_{2+\mathbf{e}_{n}}$.

Then $\operatorname{cof}_{\kappa}(x)$ has already been determined.

Case 2. $x \notin \mathfrak{A}_{2+\mathbf{e}_{n}}$.

Find the trailing term $v$ of $\left\langle T_{x}, \ell_{x}\right\rangle$.

Set $v:=\ell_{x}(v)$.

Compute nor $(\mathrm{v})$.

Then $\operatorname{cof}_{\kappa}(x)=\kappa^{\kappa} / \operatorname{nor}(\mathrm{v})$. 
Corollary 20. The algorithm described above correctly computes the cofinality of $\kappa^{\kappa} /$ wlift $_{\kappa}(x)$.

Proof. Obvious from Corollary 18 and Theorem 19.

Let us apply the algorithm to the examples of non-normal variables given in Figure 5: $\bigvee_{4}, \vee_{\omega+2}, \bigvee_{\omega^{2}}$ and $\bigvee_{\omega^{\omega^{2}}}$.

- The variable $\mathrm{V}_{4}=\mathrm{V}_{2+2}$ is highlifted from $\bigvee_{1} \oplus \mathrm{V}_{1}$ (using $\boldsymbol{\delta}_{1}^{1}$ ). Obviously, $\mathrm{V}_{1}$ is the trailing term of $\mathrm{V}_{1} \oplus \mathrm{V}_{1}$, and hence, $\operatorname{nor}\left(\mathrm{V}_{4}\right):=\mathrm{V}_{2+o\left(\mathrm{~V}_{1}\right)}=\mathrm{V}_{3}$. Therefore,

$$
\operatorname{cof}_{\kappa}\left(\mathrm{V}_{4}\right):=\kappa^{\kappa} / \text { wlift }_{\kappa}\left(\mathrm{V}_{3}\right)
$$

- The variable $\mathrm{V}_{\omega+2}$ is highlifted from $\mathrm{V}_{1} \oplus \mathrm{V}_{1}\left(\right.$ to $\left.\boldsymbol{\delta}_{3}^{1}\right)$. By the same argument, $\operatorname{nor}\left(\mathrm{V}_{\omega+2}\right)=\mathrm{V}_{\omega+1}$. Thus,

$$
\operatorname{cof}_{\kappa}\left(\mathrm{V}_{\omega+2}\right):=\kappa^{\kappa} / \text { wlift }_{\kappa}\left(\mathrm{V}_{\omega+1}\right) \text {. }
$$

- The variable $\mathrm{V}_{\omega^{2}}$ is highlifted from $\mathrm{V}_{2} \otimes \mathrm{V}_{2}$ (to $\boldsymbol{\delta}_{3}^{1}$ ) whose trailing term is $\mathrm{V}_{2}$. Therefore, $\operatorname{nor}\left(\mathrm{V}_{\omega^{2}}\right)$ is the high lift of $\mathrm{V}_{2}$ which is $\mathrm{V}_{\omega \cdot 2}$. Thus,

$$
\operatorname{cof}_{\kappa}\left(\mathrm{V}_{\omega^{2}}\right):=\kappa^{\kappa} / \operatorname{wlift}_{\kappa}\left(\mathrm{V}_{\omega \cdot 2}\right)
$$

- Finally, the variable $\mathrm{V}_{\omega^{\omega^{2}}}$ is highlifted from $\mathrm{V}_{4}$. We already computed $\operatorname{nor}\left(\mathrm{V}_{4}\right)$ earlier to be $\mathrm{V}_{3}$, so nor $\left(\mathrm{V}_{\omega^{\omega^{2}}}\right)$ is the high lift of $\mathrm{V}_{3}$ which is $\mathrm{V}_{\omega^{\omega}}$, and hence

$$
\operatorname{cof}_{\kappa}\left(\mathrm{V}_{\omega^{\omega^{2}}}\right):=\kappa^{\kappa} / \mathbf{w l i f t}_{\kappa}\left(\mathrm{V}_{\omega^{\omega}}\right)
$$

Corollary 20 and the canonicity assumption give an algorithm for computing the cofinality of any successor cardinal $\aleph_{\alpha+1}$ for $\alpha<\varepsilon_{0}$. Namely, first find the $n$ such that $\mathbf{e}_{n} \leq \alpha<\mathbf{e}_{n+1}$. Let $\alpha^{\prime}$ be such that $\alpha=\mathbf{e}_{n}+\alpha^{\prime}$. Let $x \in \mathfrak{A}_{\mathbf{e}_{n}}$ be a term with $o(x)=\alpha^{\prime}$. Let $\mathbf{v}=\operatorname{nor}(x)$. Then $\operatorname{cf}\left(\aleph_{\alpha+1}\right)=\left(\boldsymbol{\delta}_{2 n-1}^{1}\right)^{\delta_{2 n-1}^{1} / \operatorname{wlift}_{\boldsymbol{\delta}_{2 n-1}^{1}}}(\mathrm{v})=$ $\aleph_{\mathbf{e}_{n}+o(\mathrm{v})+1}$.

To illustrate the algorithm, let us compute the cofinality of $\kappa=\aleph_{\alpha+1}$ where $\alpha=\omega^{\omega^{\left(\omega^{\omega^{2}}+\omega^{\omega \cdot 2+3}\right)}}$ (so $\boldsymbol{\delta}_{5}^{1}<\kappa<\boldsymbol{\delta}_{7}^{1}$ ). Clearly, $\mathbf{e}_{2}<\alpha<\mathbf{e}_{3}$, and $\alpha^{\prime}=\alpha$ in the notation of the previous paragraph. The term $x \in \mathfrak{A}_{\mathbf{e}_{2}}$ with $o(x)=\alpha^{\prime}$ is the generator $\mathrm{V}_{\beta}$, with $\beta=\omega^{\omega^{2}}+\omega^{\omega \cdot 2+3}$. We next compute nor $\left(\mathrm{V}_{\beta}\right)$. The variable $\mathrm{V}_{\beta}$ corresponds to the high lift of the term $y=\mathrm{V}_{4} \oplus\left(\mathrm{V}_{3} \otimes \mathrm{V}_{3} \otimes \mathrm{V}_{2} \otimes \mathrm{V}_{2} \otimes \mathrm{V}_{2}\right) \in \mathfrak{A}_{\mathbf{e}_{1}}$. The trailing variable is $\mathrm{V}_{2}$, which is normal. Thus, $\operatorname{nor}\left(\mathrm{V}_{\beta}\right)=\operatorname{highlift}\left(\mathrm{V}_{2}\right)=\mathrm{V}_{\omega \cdot 2}$. So, $\operatorname{cf}(\kappa)=\boldsymbol{\delta}_{5}^{1} \boldsymbol{\delta}_{5}^{1} /$ wlift $_{\delta_{5}^{1}}\left(\mathrm{~V}_{\omega \cdot 2}\right)=\aleph_{\omega^{\omega} \omega}+o\left(\mathrm{~V}_{\omega \cdot 2}\right)+1$, whence

$$
\operatorname{cf}\left(\aleph_{\omega^{\omega}\left(\omega^{\omega^{2}}+\omega^{\omega \cdot 2+3}\right)+1}\right)=\aleph_{\omega^{\omega} \omega^{\omega \cdot 2}+1} .
$$

6.3. Computation of the Kleinberg sequences. Under the canonicity assumption, the Kleinberg sequences can now be easily read off.

Lemma 21. If $\operatorname{wlift}_{\kappa}(\mathrm{v})=\mathcal{C}_{\kappa}^{\lambda}$ is a normal ultrafilter on $\kappa:=\boldsymbol{\delta}_{2 n+1}^{1}$, then the Kleinberg sequence on $\kappa$ derived from $\mathcal{C}_{\kappa}^{\lambda}$ is given by

$$
\kappa_{n}^{\mathcal{C}^{\lambda}}:=\kappa^{\kappa} / \text { wlift }_{\kappa}(\mathrm{v} \otimes n) .
$$

Proof. Taking iterated ultrapowers as in the definition of the Kleinberg sequence corresponds to taking iterated sums: it is true in general that the $n$-fold sum ultrapower embeds into the $n$-fold iterated ultrapower (a proof can be found as [BoLö $\infty$, Proposition 13]). The upper bound for the iterated ultrapower comes from 
the Ultrapower Shifting Lemma [Lö02, Lemma 2.7] and the canonicity conjecture.

By Corollary 20, we know that the normal measures are all generated by normal variables, and by $\S 6.1$, we have a simple algorithm to compute the $o$-values of the normal variables. Therefore, we can read off the values of the Kleinberg sequences as $o(\mathrm{v}) \cdot n$ for a normal variable $\mathrm{v}$. As an example, we can read off the Kleinberg sequences on $\delta_{5}^{1}$ as follows (for $n \geq 1$ ): $\aleph_{\omega^{\omega \omega}+n+1}, \aleph_{\omega^{\omega \omega}+\omega \cdot n+1}, \aleph_{\omega^{\omega \omega}+\omega^{\omega} \cdot n+1}$, $\aleph_{\omega^{\omega}{ }^{\omega} \cdot n+1}, \aleph_{\omega^{\omega \omega+1} \cdot n+1}, \aleph_{\omega^{\omega \omega \cdot 2} \cdot n+1}$, and $\aleph_{\omega^{\omega \omega^{\omega}} \cdot n+1}$.

\section{ApPEndix}

In this appendix, we give a table (Figure 5) of all of the relevant values for our measure assignment described in $\S 5$. In this table, we give the terms and their values of $o$, ot and germ. In the next row, we list the value of $\operatorname{wlift}_{\kappa}(x)$ for some $\kappa=\boldsymbol{\delta}_{2 n+1}^{1}$ where $o(x)<\mathbf{e}_{n+1}$ (inductively, the order types of terms $x$ with $o(x)<\mathbf{e}_{n+1}$ will be $<\boldsymbol{\delta}_{2 n+1}^{1}$, so we can lift their germs to that cardinal). These first four rows of values can be computed independently of the canonicity conjecture. The following row gives the value of $\kappa^{\kappa} /$ wlift $_{\kappa}(x)$ for $\kappa=\delta_{2 n+1}^{1}$ where $o(x)<\mathbf{e}_{n+1}$ under the assumption of the canonicity conjecture. The last row lists whether the term is a normal variable or not. For the non-normal measures, we use the S-notation for the families of measures introduced in [Ja88, p. 119] (the $\tilde{\mathrm{S}}_{n}^{\ell, m}$ measure are defined as the $\mathrm{S}_{n}^{\ell, m}$ measures of [Ja88] except we use function of continuous type instead of correct type).

The table comes in two systems: the first system lists terms from $\mathfrak{A}_{\omega}$, the second system lists terms from $\mathfrak{A}_{\mathbf{e}_{2}}$. The two systems are linked by the operation of high lift: the columns in the second system correspond to those variables whose values for germ and ot are the high lifts of the terms in $\mathfrak{A}_{\omega}$ in the same column of the first system.

It is clear from the construction that all terms come with information about their stage of construction. In addition, there is some descriptive set theoretic information hidden in the recursive construction that we would like to point the reader's attention to. In constructing the measure assignment of $\S 5$, we assign the germs to the new variables in $\mathfrak{A}_{\mathbf{e}_{n+1}}$ based on the measures on terms in $\mathfrak{A}_{2+\mathbf{e}_{n}}$. One of these is slightly special: the germ assigned to $V_{2+\mathbf{e}_{n}}$ itself comes from the special variable $\mathrm{V}_{0}$ and thus is not really high lifted, but rather lifted only once. We shall say that this variable is of level $2 n+1$. All of the other newly created variables are of level $2 n+2$. This defines a notion of level for all generators of $\mathfrak{A}_{\varepsilon_{0}}$ except for $\mathrm{V}_{0}$ and $\mathrm{V}_{1}$. For example $\mathrm{V}_{2}$ is of level $1, \mathrm{~V}_{\alpha}, 2<\alpha<\omega$ are of level 2, $\mathrm{V}_{\omega}$ is of level $3, \mathrm{~V}_{\alpha}, \omega=\mathbf{e}_{1}<\alpha<\mathbf{e}_{2}=\omega^{\omega^{\omega}}$ are of level $4, \mathrm{~V}_{\omega^{\omega} \omega}$ is of level 5 , etc. This notion of level is connected to descriptive set theory in the following sense: the germs associated to variables of level $n$ are typical measures occurring in the homogeneous tree construction for the complete $\boldsymbol{\Pi}_{n}^{1}$ set.

\section{REFERENCES}

[BoLö $\infty$ Stefan Bold, Benedikt Löwe, A simple inductive measure analysis for cardinals under the Axiom of Determinacy, submitted

[Ja88] Steve Jackson, AD and the Projective Ordinals, in: A. S. Kechris, D. A. Martin, J. R. Steel (eds.), Cabal Seminar 81-85, Proceedings, Caltech-UCLA Logic Seminar 1981-85, Springer-Verlag 1988 [Lecture Notes in Mathematics 1333], p. 117-220 


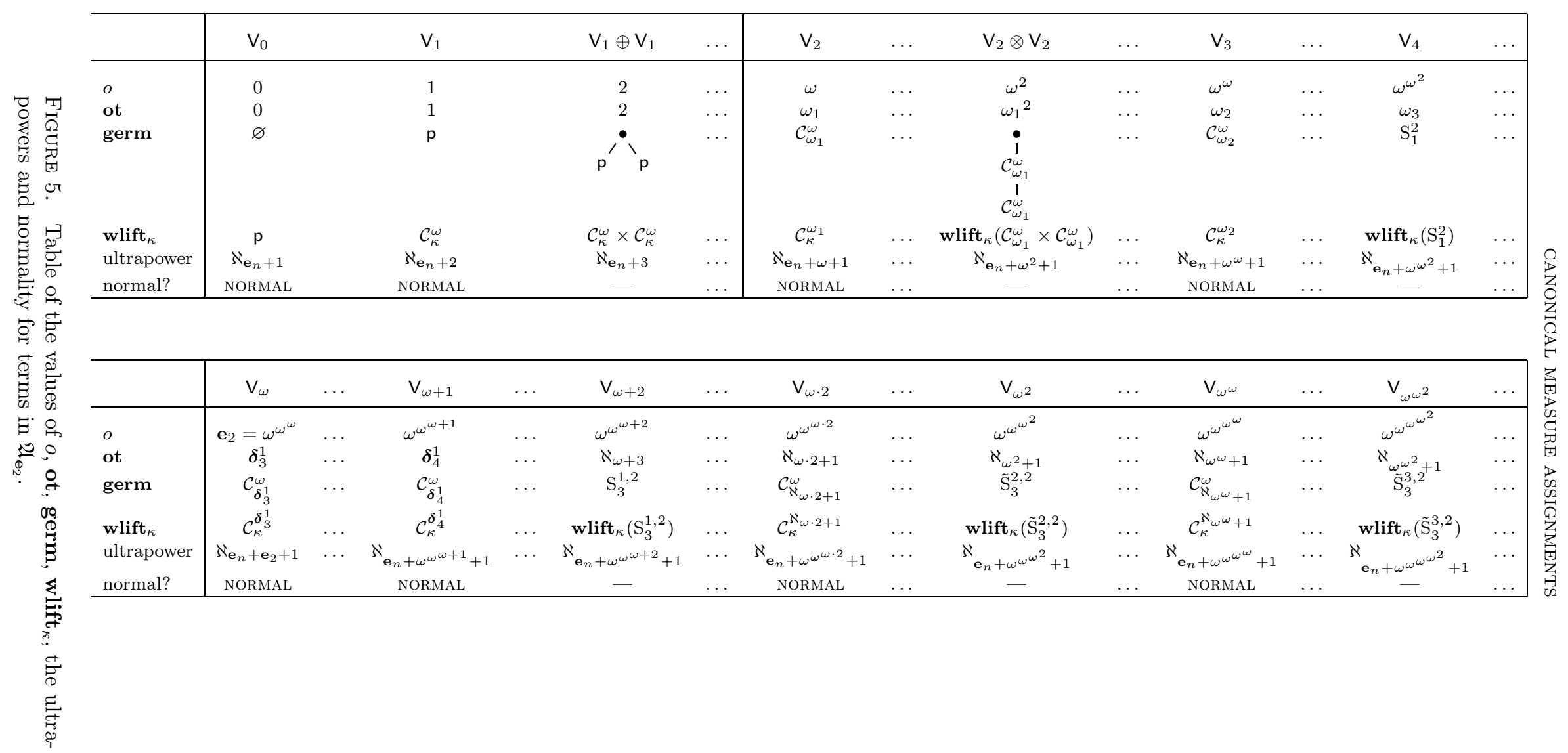


[Ja99] Steve Jackson, A Computation of $\boldsymbol{\delta}_{5}^{1}$, Memoirs of the American Mathematical Society 140 (1999), viii+94 pages

$[\mathrm{JaKh} \infty]$ Steve Jackson, Farid T. Khafizov, Descriptions and Cardinals below $\boldsymbol{\delta}_{5}^{1}$, accepted for publication in Journal of Symbolic Logic

$[\mathrm{Ja} \infty]$ Steve Jackson, Structural Consequences of $\mathrm{AD}$, to appear in: M. Foreman, A. Kanamori, M. Magidor (eds.), Handbook of Set Theory

[Ka94] Akihiro Kanamori, The Higher Infinite, Large Cardinals in Set Theory from Their Beginnings, Springer-Verlag 1994 [Perspectives in Mathematical Logic]

[Ke78] Alexander S. Kechris, AD and Projective Ordinals, in: A. S. Kechris, Y. N. Moschovakis, Cabal Seminar 76-77, Proceedings, Caltech-UCLA Logic Seminar 1976-77, Springer-Verlag 1978 [Lecture Notes in Mathematics 689], p. 91-132

[Kl77] Eugene M. Kleinberg, Infinitary Combinatorics and the Axiom of Determinateness, Springer-Verlag 1977 [Lecture Notes in Mathematics 612]

[Lö02] Benedikt Löwe, Kleinberg Sequences and partition cardinals below $\boldsymbol{\delta}_{5}^{1}$, Fundamenta Mathematicae 171 (2002), p. 69-76.

[Mo80] Yiannis N. Moschovakis, Descriptive Set Theory, North-Holland 1980 [Studies in Logic and the Foundations of Mathematics 100]

Department of Mathematics, University of North Texas, Denton, P.O. Box 311430 , TX 76203-1430, U.S.A.

E-mail address: jackson@unt.edu

Institute for Logic, Language and Computation, Universiteit van Amsterdam, Plantage Muidergracht 24, 1018 TV Amsterdam, The Netherlands

E-mail address: bloewe@science.uva.nl 